\title{
Carbon Interstitial in the Diamond Lattice
}

\author{
C. Weigel*, D. Peak, and J. W. Corbett ${ }^{\dagger}$ \\ Physics Department, State University of New York at Albany, Albany, New York 12222
}

G. D. Watkins and R. P. Messmer

General Electric Corporate Research and Development, Schenectady, New York 12301

(Received 20 February 1973)

\begin{abstract}
Linear-combination-of-atomic-orbitals-molecular-orbitals cluster calculations using the extended Hückel theory are carried out for the interstitial carbon in the diamond lattice. The results suggest that the interstitial configuration is not the tetrahedral or hexagonal site, as has been previously assumed, but is instead an "interstitialcy" configuration, i.e., either a split- $\langle 100\rangle$ interstitial (which our results favor) or a bond-centered interstitial. The predicted minimum-energy configuration changes with charge state, suggesting that the interstitial in the diamond lattice is a possible example of the Bourgoin mechanism of athermal migration of a defect in the presence of ionizing radiation.
\end{abstract}

\section{INTRODUCTION}

The problem of treating the electronic states of defects and impurities in semiconducting covalent solids is one of great importance, both from a fundamental and a practical point of view. As has been discussed by Messmer and Watkins, ${ }^{1-3}$ there is no general theoretical description which has achieved even moderate success for these problems. They introduced a molecular cluster approach to this problem, and as a first step used a particularly simple molecular-orbital (MO) approximation, the extended Huickel theory (EHT). As they discussed, this approach provides wave functions, locates energy levels with respect to band edges, is amenable to treating lattice distortions and relaxations around the defect, and provides the basis for a practical computational scheme. They argued that the utilization of more sophisticated molecular-orbital theories would be desirable, but that in view of the computational complexities, the use of EHT is desirable as a survey tool to treat a number of problems that heretofore have not been fully treated.

Here we use the Messmer and Watkins approach to treat the problem of the interstitial in the diamond lattice. This is a problem in which experiment has been very much in need of support from theory. As is well documented, in silicon, for example, EPR studies have identified the vacancy ${ }^{4}$ (one of the problems considered by Messmer and Watkins) and a number of vacancy-related defects. ${ }^{5}$ However, although there are a number of unidentified EPR spectra, none have been firmly established as belonging to an interstitial. We will return to the question of experimental evidence, and other theoretical work in Sec. VI. In Sec. II, we discuss EHT as applied to this interstitial problem. In Sec. III, we present calculations of the energy of an interstitial atom as it is moved from site to site in an otherwise static lattice, i.e., a lattice in which the lattice atoms remain fixed. These calculations show, as has been described in a preliminary report, ${ }^{6}$ that neither the tetrahedral interstitial site nor the hexagonal interstitial site correspond to potential-energy minima; in fact, the tetrahedral site is a potential-energy maximum and the hexagonal site a saddle point. This result was surprising since the earlier theoretical work ${ }^{7-12}$ on the interstitial had assumed that one of these sites would be the minimum-energy interstitial configuration. Instead, it is found that the potential energy of the interstitial is lowest near the lattice atoms, indicating that the interstitial participates in substantial bonding with the lattice.

In Sec. IV, we present calculations in which some relaxation of lattice atoms is permitted. It is found that the lowest-energy configuration corresponds to a split- $\langle 100\rangle$ interstitial, with the bond-centered interstitial very little higher in energy for the neutral interstitial atom. In Sec. V, we consider the electrical levels of the various interstitial configurations. In Sec. VI, we discuss the utility of these calculations, their limitations and implications.

\section{THEORY}

Recently, a number of semiempirical theoretical approaches to molecular problems were developed (cf. Ref. 13). These are typically approximations to the more rigorous Hartree-FockRoothaan self-consistent-field (SCF) theory ${ }^{14-16}$-itself often inadequate-and are justified by their utility in, say, giving physical insight into the problem, in explaining trends, etc.

We employ here such a semiempirical theorythe extended Hückel theory. ${ }^{17}$ EHT may be applied at the present time to systems containing up to some 300 atomic orbitals on the typically available modern computers. With the possible exception of 
the complete-neglect-of-differential-overlap (CNDO) method, ${ }^{18,19}$ there are no other methods available at present with the simplicity and computational speed needed to handle clusters of atoms as large as the systems we wish to investigate. CNDO, moreover, does not seem to represent the band structure of homopolar crystals nearly as well as EHT. ${ }^{20}$

EHT has been widely employed and for a number of problems gives good results; among them are the following: barriers to internal rotation in certain molecules like $\mathrm{C}_{2} \mathrm{H}_{6}, \mathrm{C}_{3} \mathrm{H}_{8},{ }^{17} \mathrm{~N}_{2} \mathrm{O}_{4}$, or $\mathrm{B}_{2} \mathrm{Cl}_{4}{ }^{21}$; vibrations in molecules like $\mathrm{Cu}_{2}{ }^{22}$; deep impurity levels in diamond ${ }^{23}$; Jahn-Teller distortions in diamond ${ }^{23}$ or in the $\mathrm{CuCl}_{6}{ }^{4-}$ radical. ${ }^{24}$

Especially relevant to our calculations is the fact that EHT can give reasonable predictions for the following: elastic properties of diamond ${ }^{23}$ and of graphite ${ }^{25}$; stability of defects in graphite ${ }^{25}$; defect migration and formation energies in graphite $^{25}$; configurations of molecules like methane, ethane, propane, acetylene, ethylene,${ }^{17}$ transbutadiene, benzene, ${ }^{26} \mathrm{~F}_{2} \mathrm{O}$, or $\mathrm{BH}_{2}{ }^{-} .{ }^{27}$

But when using EHT, one should bear in mind the following examples of limitations or failures. EHT can be applied justifiably only to systems with uniform charge densities, since it is not a self-consistent method. As previously pointed out, ${ }^{28}$ EHT may give unreliable predictions for ionic systems, where the electronegativity difference between adjacent atoms exceeds 1.3 on the Pauling $^{29}$ or 1.0 on the Sanderson scale. ${ }^{30}$ Also EHT gives wrong isomerization and strain energies in certain cases like pentanes or hexanes. ${ }^{17}$ For some systems like $\mathrm{H}_{2}, \mathrm{Li}_{2}, \mathrm{Be}_{2}, \mathrm{C}_{2}, \mathrm{HF}$, and $\mathrm{HCl}$, EHT yields no minimum as the internuclear distance is changed. ${ }^{31}$ The results are also completely unrealistic for small internuclear distances.

The mathematical origins and approximations of EHT have been discussed by Newton, Boer, and Lipscomb $^{32}$ and Moore. ${ }^{33}$

The EHT equations for the molecular-orbital (MO) energies and MO coefficients have the same form as the more rigorous Hartree-Fock-Roothaan equations, ${ }^{16}$ viz.,

$$
\sum_{\nu=1}^{n}\left(H_{\mu \nu}-E_{i} S_{\mu \nu}\right) c_{\nu i}=0, \quad \mu=1, \ldots, n
$$

The $c_{\nu i}$ 's are MO coefficients given by

$$
\phi_{i}=\sum_{\nu=1}^{n} c_{\nu i} \chi_{\nu}
$$

where the $\chi_{\nu}$ 's are the atomic and the $\phi_{i}$ 's the molecular orbitals. The $S_{\mu \nu}$ are overlap matrix elements.

The $H_{\mu \nu}$ are elements of an effective one-electron Hamiltonian given by

$$
H_{\mu \nu}=\frac{1}{2} K\left(H_{\mu \mu}+H_{\nu \nu}\right) S_{\mu \nu}, \quad \mu \neq \nu .
$$

This is the so-called Wolfsberg-Helmholtz form ${ }^{34}$ of the Mulliken approximation ${ }^{35}$ for the $H_{\mu \nu}$ 's.

The diagonal elements are taken to be

$$
H_{\mu \mu}=-I_{\mu},
$$

with $I_{\mu}$ the empirical atomic ionization energies. The constant $K$ in Eq。(3) is determined empirically, ranging between 1 and 2 ; we have used $1.75 .^{17}$

Equations (1)-together with the normalization conditions for the $\phi_{i}$ 's-yield the MO coefficients $c_{\nu i}$ and the MO energies $E_{i}$.

We use as atomic orbitals one $2 s$ and three $2 p$ Slater orbitals on each carbon atom; the orbital exponent is chosen equal 1.625 for all atoms. ${ }^{19}$ The $I_{\mu}$ 's used are $19.44 \mathrm{eV}$ and $10.67 \mathrm{eV}$ for the $2 s$ and $2 p$ orbitals, respectively. ${ }^{19}$ These parameters comprise our "standard" set of parameters.

In EHT, the total energy of a system is approximated by

$$
E_{\mathrm{EHT}}=\sum_{i} n_{i} E_{i}
$$

where $n_{i}$ is the occupation number of the level $E_{i}$.

To be sure, this is only an approximation to the total energy $E_{T}$ of a system. As Slater ${ }^{36}$ pointed out, the energy $\sum_{i} n_{i} E_{i}$ as obtained from HartreeFock equations (to which EHT is an approximation) is equal to $E_{T}$ minus the repulsion energy of the nuclei, $V_{\mathrm{NN}}$, plus the repulsion energy of the electron pairs, $V_{\text {ee }}$. Slater observed that, in a very rough approximation, $V_{\mathrm{NN}}$ canceled $V_{\text {eu }}$, so that $\sum_{i} n_{i} E_{i}$ displayed some sort of parallel behavior to $E_{T}$; Slater investigated the binding energies of $X_{2}$ molecules $(X=\mathrm{Be}, \mathrm{B}, \mathrm{C}, \mathrm{N}, \mathrm{O}, \mathrm{F})$. (Later Goodisman ${ }^{37}$ stated that it was not $V_{\theta e}$ that roughly equaled $V_{\mathrm{NN}}$ but rather $\Delta V_{\mathrm{ee}}=V_{\mathrm{ee}}^{m}-\sum_{a} V_{\mathrm{ee}}^{a}$, where $a$ stands for the atoms that form the molecule $m_{\text {. }}$ ) Similarly, the EHT binding energies, as calculated for boron hydrides ${ }^{38,39}$ and hydrocarbons, ${ }^{17}$ are systematically larger than the experimental values by a factor of 1.8 , but are otherwise internally consistent. Another parallel behavior of EHT energies to $E_{T}$ was observed by Allen and Russell, ${ }^{27}$ when they varied the bond angle in homopolar systems.

It was stressed by these authors, as well as by others, e.g. , Blyholder and Coulson, ${ }^{26}$ that this parallel behavior of $E_{\mathrm{EHT}}$ to $E_{T}$ can only be expected for systems with reasonably uniform charge distributions.

In view of all these observations, we expect our results for carbon interstitials in diamond to be useful in showing trends and qualitative behavior, although $E_{\mathrm{EHT}}$ is a poor approximation to $E_{T}$ itself, a point we will return to in Sec. VI。 


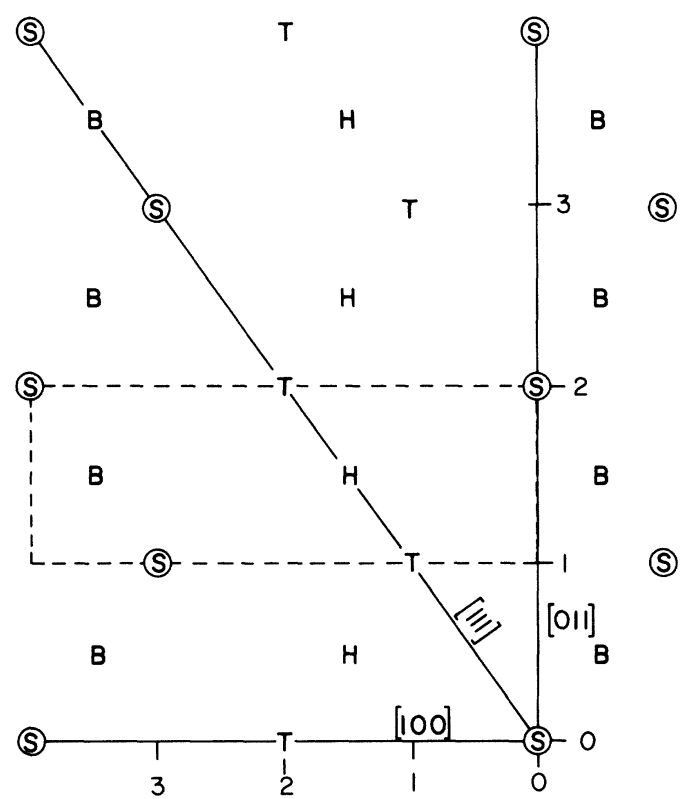

FIG. 1. (01i) plane in the diamond lattice. This plane contains the major symmetry axes and all the special symmetry points: substitutional atom sites $(S)$; bondcenter sites $(B)$, i. e., half-way between adjacent substitutional sites; tetrahedral interstitial sites $(T)$; hexagonal interstitial sites $(H)$. The coordinates along the [100] and [011] axes allow for identification of specific sites.

\section{STATIC LATTICE}

\section{A. Standard Parameters}

All our calculations were carried out on diamondlattice-model crystals. The diamond lattice belongs to the space group ${ }^{40} F d 3 m$ (Schoenfliess notation $\left.O_{h}^{7}\right)$. Figure 1 shows a (011) plane containing all major symmetry and principal crystal axes. In terms of that group, the several sites of importance to us are labeled unambiguously: the substitutional $(S)$ site, the tetrahedral interstitial $(T)$ site, the bond-centered $(B)$ site, and the hexagonal interstitial $(H)$ site. In terms of the coordinates in Fig. 1, $(S)$ sites can be found at $(0,0,0),(4,0,0)$, or $(0,2,2) ;(T)$ sites at $(2,0,0),(1,1,1)$, or $(2,2,2)$. The $(B)$ site is half-way between lattice atoms, e.g., at $(-0.5,0.5,0.5)$ or $(3.5,1.5,1.5)$, whereas the $(H)$ site is the center of a "chair-shaped" sixmembered ring $^{41}$ at $(1.5,0.5,0.5)$ or $(1.5,1.5,1.5)$. $(S)$ and $(T)$ sites have point group symmetry $\overline{4} 3 m\left(T_{d}\right) ;(B)$ and $(H)$ sites have $\overline{3} m\left(D_{3 d}\right)$.

The calculation for a lattice (with no interstitial atom) consisting of 30 carbon atoms results in the energy levels shown in Fig. 2(a). As can be seen, these 120 MO levels can be divided into two bands, a valence and a conduction band. The energy gap between the bands is a little more than $10 \mathrm{eV}$, while the observed gap is $5.5 \mathrm{eV} .^{42}$ This discrep- ancy is attributed to the finite size of the cluster. ${ }^{23}$ The gap width decreases with increasing number of atoms in a cluster; it is $9.5 \mathrm{eV}$ for a 35-atom cluster, $9.1 \mathrm{eV}$ for a 47-atom cluster, and $7.4 \mathrm{eV}$ for a 71-atom cluster. ${ }^{1}$ EHT band-structure calculations, ${ }^{43}$ using the same set of parameters, i.e., calculations for the infinite cluster, yield a band gap of $4.7 \mathrm{eV}$.

There are more levels in the valence band than in the conduction band, because the energies for all the 40 dangling orbitals on the surface of the cluster are also in this band. The remaining 80 levels are divided equally between valence and conduction bands.

To obtain the energy $E_{\mathrm{EHT}}[\mathrm{Eq}$. (5)], all of the valence-band levels are filled with two electrons (a total of 160) and their respective one-electron energies are summed up. By doing so, one can obtain the elastic properties of the crystal. ${ }^{3,23} \mathrm{~A}$ more complete discussion of this procedure is provided elsewhere. ${ }^{1}$

The center of this 30 -atom diamond lattice is a tetrahedral $(T)$ site. Placing an interstitial car-

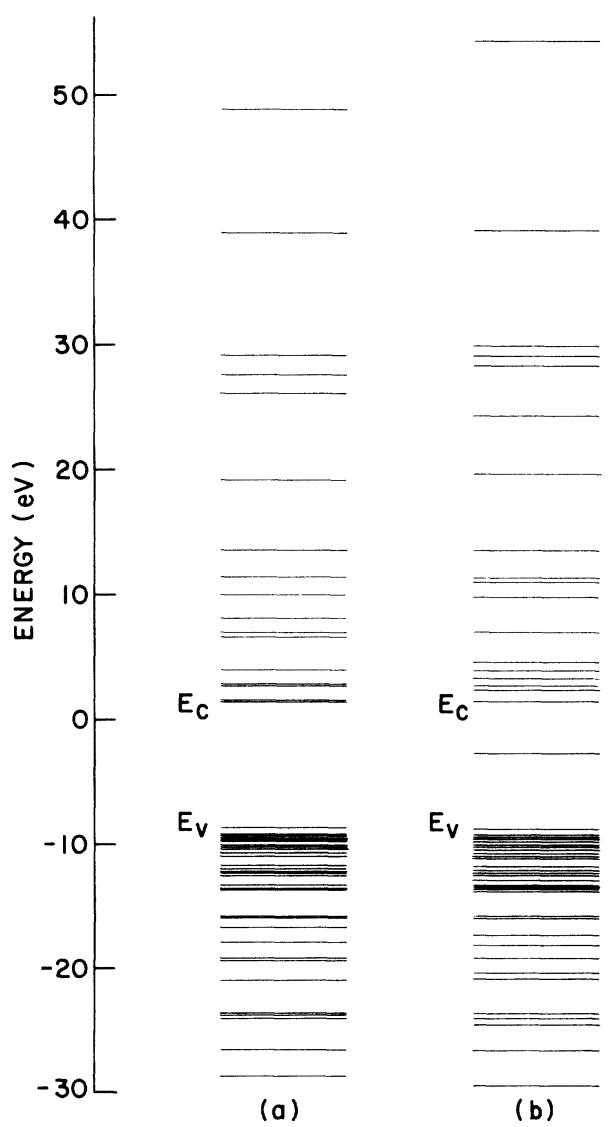

FIG. 2. (a) Energies of the one-electron MO's for the 30-carbon-atom "diamond." (b) Energies of the same model crystal with an additional carbon atom at the central tetrahedral interstitial site. 


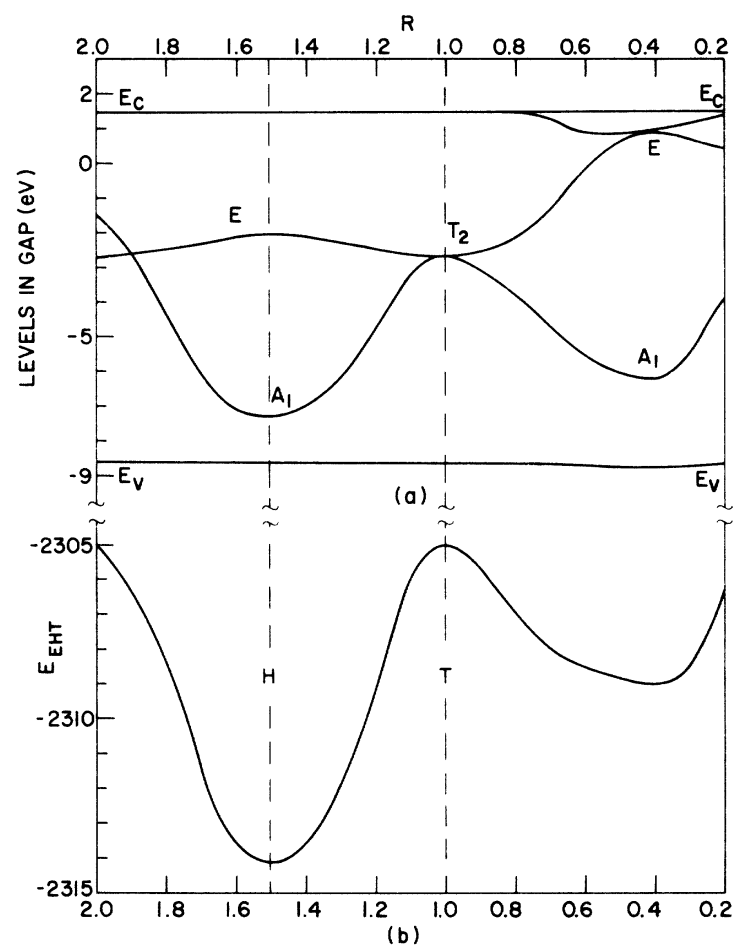

FIG. 3. (a) Electronic energies and (b) $E_{\mathrm{EHT}}$ energy (for the neutral charge state) of the 30-carbon-atom diamond with the interstitial atom moved along the [111] axis containing the central tetrahedral interstitial position $(R=1.0)$. The coordinates are to be understood in terms of Fig. 1. The symmetry of the energy levels is shown; the interstitial in this axis of the 30-atom diamond has $T_{d}$ point group symmetry at $R=1.0, C_{3 v}$ otherwise. For the neutral charge state the $T_{2}$ level holds four electrons.

bon atom in this position while keeping all other atoms in their normal positions results in the energies shown in Fig. 2(b).

Again, the valence band contains 80 levels. But now there is a defect level in the gap with the band edges essentially unchanged. This level is threefold degenerate. The fourth level originating from the interstitial carbon is in the conduction band. Since the neutral carbon atom has four valence electrons, we put four more electrons into this new set of energy levels; thus, 82 levels in total are occupied with two electrons each.

As the interstitial carbon leaves the $(T)$ site, the symmetry of the crystal is lowered and therefore the degeneracy of the levels in the gap is lifted. This can be observed in Fig. 3, which shows the dependence of band edges, impurity levels, and $E_{\mathrm{EHT}}$ for a traverse of the interstitial along the [111] axis of Fig. 1.

For an infinite lattice, the energies at $R=2$ should be the same as at $R=1$, since the two sites would be equivalent [both are $(T)$ sites]. The rea-
TABLE I. Various model clusters used in calculations (without interstitials).

\begin{tabular}{cccc}
\hline \hline Symbol & Model center & $\begin{array}{c}\text { No. of atoms } \\
\text { in cluster }\end{array}$ & $\begin{array}{c}\text { No. of levels in } \\
\text { valence band }\end{array}$ \\
\hline T30 & Tehrahedral site & 30 & 80 \\
B32 & Center of bond & 32 & 85 \\
S35 & Substitutional site & 35 & 88 \\
S47 & Substitutional site & 47 & 124 \\
\hline \hline
\end{tabular}

Son that they are not is that the $R=2$ site is already near the surface of the model cluster used, and the corresponding levels are changed due to surface effects. To avoid these surface effects in subsequent calculations, we always chose clusters such that the interstitial was never too near the surface; to be specific, the model clusters listed in Table I were used.

As can be seen from the table, the number of levels in the valence band varies from cluster to cluster; hence, the value of $E_{\mathrm{EHT}}$ of the system is also different. But corresponding energy plots for different clusters always displayed the same qualitative behavior, so that it was possible to fit judiciously these different equivalent plots to one another. This procedure is illustrated for the traverse along a [011] axis containing the $(S)$ sites in Fig. 4. The $E_{\mathrm{E} \mathrm{HT}}$ energies were obtained for other paths in the same way.

The energy contours were plotted (see Fig. 5) in the part of the (011) plane which is enclosed by the dashed line in Fig. 1; here we have followed the lead of comparable work in the metals, e.g. , Johnson and Brown. ${ }^{44}$ The notation in Fig. 5 is the

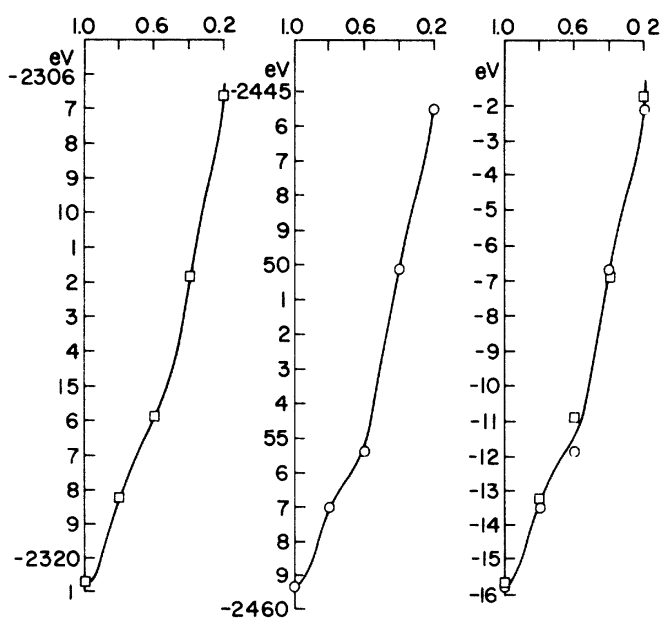

(a)

(b)

(c)

FIG. 4. Method of combining results from different lattices illustrated for claculations along the [011] axis (a) in the $T 30$ lattice and (b) in the $B 32$ lattice which give the adjusted results in (c), to be used in Fig. 5 . The coordinates are to be understood in terms of Fig. 1. 


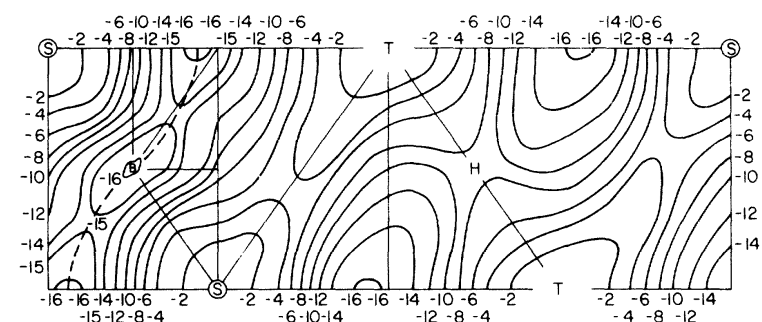

FIG. 5. Potential energy (eV) contours for the neutral carbon interstitial in the static diamond lattice calculated with the standard parameters. The dashed line indicates the migration path.

same as in Fig. 1. The rectilinear solid lines indicate axes, along which calculations have actually been carried out. The rest of the plot was arrived at by connecting points of equal energy values, considering continuity and symmetry arguments (e.g. , symmetry requires contours to cross $\langle 100\rangle$ axes at right angles, etc. ). The energies are given in units of $\mathrm{eV}$, the numbers representing a relative scale, the highest energy [at the $(T)$ site] being set to zero (cf. Fig. 5).

This energy contour map for the static lattice is not to be taken as a final result, but rather as a first approximation, especially in the region near the $(S)$ and $(B)$ sites where the energy contours are expected to change considerably, when lattice relaxations are allowed. The region near the $(T)$ and $(H)$ sites, however, is relatively distant from atoms and can therefore be expected to keep its characteristic features even in a distorted lattice.

Among the notable features of Fig. 5 is a local energy maximum for the tetrahedral $(T)$ site and a saddle point for the hexagonal $(H)$ site. All theoretical treatments of the interstitial up to $1971^{7-12}$ had assumed that one of these sites was the lowestenergy site for the interstitial. From our calculations, there are two minima of comparable energy: the bond-center $(B)$ site and a site on the [100] axis near $(3,2,2)$ (see Fig. 1).

In Table II a comparison is made for three specific lattice positions, as they are computed in different clusters and shown in the contour map. The highest energy is always set to zero. As indicated earlier, the discrepancies are due to the fact that surface effects influence the results for a specific lattice site differently in different clusters. The contour-map values for the $(T)$ and $(H)$ sites as well as their vicinity originate from the $T 30$ lattice, while the region around $(B)$ was taken from the $B 32$ lattice, the reason for this procedure being the same as was pointed out earlier. In any event, the results are consistent, as far as relative magnitude is concerned.

These results argue that an interstitial atom will not be found at $(T)$ or $(H)$, but rather it will go to
TABLE II. Relative energies (eV) of several sites as evaluated in the static lattice calculations on the various model lattices and as shown in Fig. 5.

\begin{tabular}{lrrrrc}
\hline \hline \multicolumn{4}{c}{ Site } & \multicolumn{4}{c}{ Model-1attice type } & Values used in \\
& $T 30$ & \multicolumn{1}{c}{$B 32$} & \multicolumn{1}{c}{$S 35$} & \multicolumn{1}{c}{$S 47$} & \multicolumn{1}{c}{ Fig. 5. } \\
Tetrahedral & 0.0 & 0.0 & 0.0 & 0.0 & 0.0 \\
Hexagonal & -9.1 & -9.3 & -10.6 & -9.1 & -9.1 \\
Bond center & -14.5 & -15.5 & -18.8 & -15.2 & -16.1 \\
\hline
\end{tabular}

the region near $(S)$ or $(B)$.

\section{B. Band-Structure Parameters}

Messmer ${ }^{43}$ used EHT with the same parameters as in our calculations to obtain the band structure of the infinite diamond lattice. He showed that his results could be improved considerably when two parameters were changed, viz., the Slater orbital exponent for $2 s$ orbitals from 1.625 to 2.0 and the empirical constant $K$ from 1.75 to 1.575 for $2 s-2 p$ interactions.

To check the sensitivity of our results to these parameters, we carried out the static lattice calculations with this new set of parameters. As Messmer pointed out, the change in the parameters has no dramatic effect on the valence band. Since it is essentially the valence-band energies that contribute to the energy $E_{\mathrm{EHT}}$, no important difference in the energy-contour map was expected. The results are shown in Fig. 6.

Examining the energy contours in Fig. 6, one finds again a local energy maximum for the $(T)$ site and a saddle point for the position $(H)$. Moreover, the two minima are observed, the lowest energy is obtained for the $(B)$ site, while the energy minimum lying in the [100] axis is about $1.5 \mathrm{eV}$ higher. It is important to note that the new parameters still yield minima, saddle points, or maxima where there were minima, etc. for the calculations with the original set of parameters.

Table III compares the results for the $(T),(B)$, and $(H)$ sites with each other, which can be compared to the previous results in Table II. Again, the highest energy is set to zero. These results

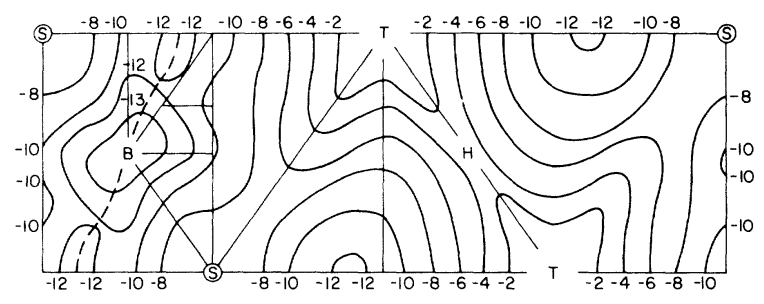

FIG. 6. Potential energy (eV) contours for the neutral carbon interstitial in the static diamond lattice calculated with the band-structure paramteres. The dashed line indicates the migration path. 
TABLE III. Relative energies (eV) of several sites as evaluated in the static lattice calculations on the various model lattices using the band-structure parameters. Compare with Table II which uses the standard parameters.

\begin{tabular}{|c|c|c|c|c|}
\hline \multirow[b]{2}{*}{ Site } & \multicolumn{3}{|c|}{ Model-lattice type } & \multirow{2}{*}{$\begin{array}{l}\text { Values used in } \\
\text { Fig. } 6\end{array}$} \\
\hline & $T 30$ & B32 & $S 35$ & \\
\hline Tetrahedral & 0.0 & 0.0 & 0.0 & 0.0 \\
\hline Hexagonal & -2.7 & -2.9 & -4.0 & -2.7 \\
\hline Bond-centered & -12.2 & -12.9 & -13.8 & -13.7 \\
\hline
\end{tabular}

show consistency with previous results, the same ordering occurring versus energy.

For our purposes the new parameters do not give essentially new results. Furthermore, they could not reproduce the elastic lattice constants ${ }^{45}$ as well as the old parameters. ${ }^{23}$ Since we are interested in finding defect configurations, we prefer a set of parameters that gives a good fit to elastic properties. Hence, in the following calculations, the standard set of parameters was used.

\section{SPLIT INTERSTITIALS AND LATTICE RELAXATIONS}

The next stage in our calculations, following the static lattice calculations, is to allow one lattice atom to relax with the interstitial to form a split interstitial, the rest of the model crystal being held static. A "split interstitial" is defined as a substitutional atom replaced by two atoms symmetrically displaced with respect to the original substitutional site. Our nomenclature conforms to that common in the metals (see, for example, Ref. 46) where the interstitials are also thought to have split configurations. If these two atoms are located along the $\langle 100\rangle$ axis, the configuration is called a split- $\langle 100\rangle$ interstitial, etc. (see Fig. 7)。

The split-interstitial calculations have been carried out in lattices centered on a substitutional
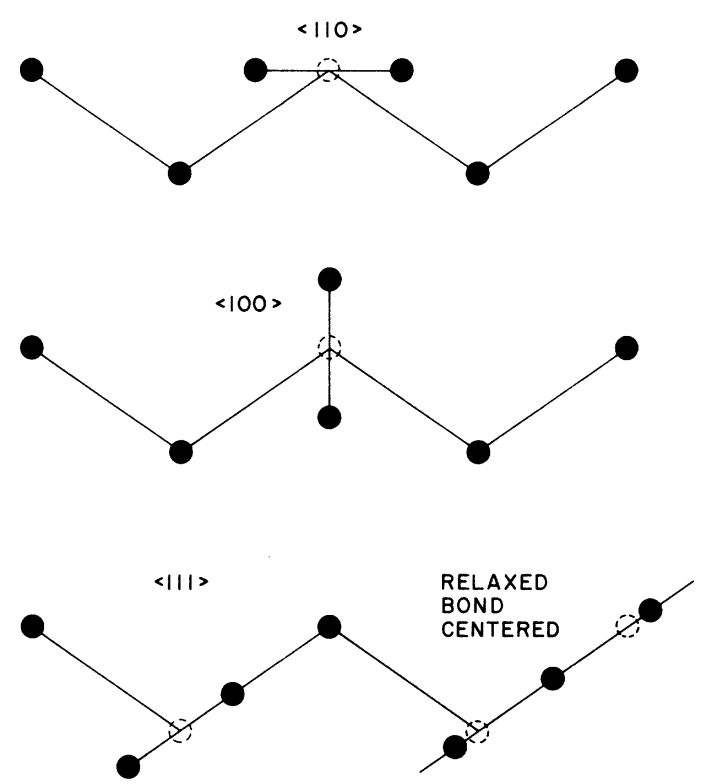

FIG. 7. Split interstitials, i.e., a pair of atoms sharing a substitutional atom site (dashed circles), of different axial orientations, and the relaxed bondcentered configuration; all shown in a $\{110\}$ plane.

$(S)$ site, first in the $S 35$ lattice, then-with the more distant neighbor relaxations in mind-in the $S 47$ lattice. The positions of the split-interstitial atoms were symmetrically changed until a minimum of $E_{\mathrm{EHT}}$ was obtained. It was found that the spatial separation between the two split-interstitial atoms at the respective minimum energies were the same in the $S 35$ and $S 47$ lattices. These configurations are shown in Fig. 7. Also, the same ordering according to energy between the various split interstitials was obtained.

In Table IV, the $E_{\mathrm{EHT}}$ energies corresponding to the mentioned configurations as well as those of single interstitials at some specific lattice sites

TABLE IV. $E_{\text {EHT }}$ energies (eV) of the various interstitial types calculated (using standard paramters) in the $S 47$ lattice.

\begin{tabular}{|c|c|c|c|c|}
\hline Interstitial type & $\begin{array}{c}\text { Relative } \\
\text { energy }\end{array}$ & $\begin{array}{c}\text { Energy difference } \\
\text { between relaxed } \\
\text { and } \\
\text { static case } \\
\end{array}$ & $\begin{array}{l}\text { Number } \\
\text { of } \\
\text { nearest } \\
\text { neighbors }\end{array}$ & $\begin{array}{c}\text { Amount of } \\
\text { relaxation } \\
(\text { bond length }=1)^{2, b}\end{array}$ \\
\hline$\langle 100\rangle$ split $^{\mathrm{c}}$ & -19.8 & -0.9 & 4 & +0.05 \\
\hline$\langle 110\rangle$ split $^{\mathrm{c}}$ & -16.9 & $(-1.1)$ & 2 & $(+0.15)$ \\
\hline$\langle 111\rangle$ split $^{\mathrm{c}}$ & -17.2 & ... & ... & .. \\
\hline Relaxed bond-centered ${ }^{d}$ & -18.6 & $\cdots$ & $\cdots$ & $\cdots$ \\
\hline Tetrahedral $^{c}$ & 0.0 & $(-2.7)$ & 4 & $(+0.25)$ \\
\hline Hexagonal $^{c}$ & -9.1 & $(-0.5)$ & 6 & $(+0.1)$ \\
\hline
\end{tabular}

${ }^{a}$ Values in brackets result from relaxation calculations carried out in lattices other than S47; cf. text.

$b_{+}+$sign for the amount of relaxation indicates relaxation away from the interstitial configuration.

${ }^{c}$ Calculated in the otherwise undistorted lattice.

${ }^{\mathrm{d}}$ Two nn's of bond-centered atom relaxed; cf. Fig. 7 . 
are listed for the $S 47$ lattice. As can be seen, all split interstitials and the relaxed bond-centered configuration have considerably lower energies than the single interstitials. Among the split interstitials, the $\langle 100\rangle$ one is the lowest. (The values of the relative energies given in Table IV are slightly different from those in Ref. 6, which were based on calculations in the $S 35$ lattice. Also, it should be noted that similar calculations have been carried out with the "band-structure parameters"; they yielded, in general, the same split-interstitial configurations and ordering.)

These results do not depend on which model crystals ( $\$ 35$ or $S 47$ ) are used for the calculations. They indicate that split-interstitial and relaxed bond-centered configurations must be taken into consideration, since they are energetically favored in comparison to single interstitials.

It should be noted that Watkins ${ }^{47}$ recognized rather early the possibility of the bond-centered configuration and Friedel ${ }^{48}$ discussed qualitatively the split- $\langle 100\rangle$ interstitial, but Watkins et al ${ }^{6}$ first showed the importance of these low-energy configurations, previous treatments of the interstitial having assumed the $(T)$ or $(H)$ site, as mentioned in Sec. III A. Singhal ${ }^{49}$ has subsequently reported calculations based on the solid-state scattering theory indicating that the $(B)$ site is also much more probable for the Si interstitial in the Si lattice than the $(T)$ site. These latter calculations did not consider lattice relaxations, however.

We next consider further lattice relaxations in our model calculations. In doing so, we hold a given interstitial configuration of the type previously considered fixed and let the next shell of equivalent neighbors (nn's) move back or forth the same distance along an axis pointing to the center of the interstitial configuration. To avoid unnecessary complications, these axes were always taken to be $\langle 111\rangle$ axes.

The nn relaxations for single interstitials were considered first. The calculations for the single interstitial at the $(T)$ and $(H)$ sites were carried out in the $T 30$ lattice; the extent of the nn relaxations as well as the energy gained by the relaxations can be found in Table IV. These numbers may be slightly different for the $S 35$ or $S 47$ lattices; but their values indicate $[-2.7 \mathrm{eV}$ for $(T)$, $-0.5 \mathrm{eV}$ for $(H)]$ that the single interstitials even with nn relaxations have considerable higher energies (about $10 \mathrm{eV}$ ) than split interstitials without $\mathrm{nn}$ relaxations.

The split- $\langle 100\rangle$ interstitial is the most interesting, for it seems to be lowest in energy. The $\mathrm{nn}$ relaxations, as calculated in the $S 35$ lattice, yielded spurious results (the energy monotonically decreased as the four nn's were relaxed). This was attributed to the finite size of the $S 35$ lattice, where the four nn's were essentially surface atoms. In a larger model cluster, the $S 47$ lattice, a minimum in $E_{\mathrm{EHT}}$ was obtained, about $0.9 \mathrm{eV}$ lower than for the unrelaxed case. This configuration may be close to the final one within the theoretical framework we have employed, as subsequent symmetric variation of the positions of the two splitinterstitial atoms (the four nn's being kept at their relaxed positions) did not yield a lower energy.

At this stage it would seem logical to include relaxations of the six nn's of the relaxed bond-centered configuration, especially since this configuration comes closest in energy to the $\langle 100\rangle$ split. But even in the large $\$ 47$ lattice, three of these $n n$ 's are essentially surface atoms. With the experience we had with the $\langle 100\rangle$-split calculations mentioned above, we were reluctant to do these calculations and have deferred them for a possible later study using a larger lattice. We will also not consider $\mathrm{nn}$ relaxations for the $\langle 111\rangle$ split.

For the $\langle 110\rangle$ split, which has the highest energy among the split interstitials in the unrelaxed case, the relaxation of the two equivalent nn's resulted in an energy lowering of $1.1 \mathrm{eV}$ for the new minimum (as calculated in the $S 35$ lattice). This is an indication that the energy difference between the $\langle 100\rangle-$ and $\langle 110\rangle$-split interstitials may be practically the same with or without the inclusion of $n n$ relaxations.

\section{ELECTRONIC ENERGY LEVELS}

One major advantage of EHT is that it gives not only $E_{\mathrm{EHT}}$, a measure of the total energy of the system, but also all the individual electronic energy levels. Of course, in the model crystals we are using, we will not obtain the $10^{22}$ or so energy levels corresponding to the solid, but we do obtain localized energy levels corresponding to the defect and levels corresponding to the valence and conduction bands. This was already shown in Figs. 2 and 3 for the carbon interstitial located in a [111] axis of the T30 lattice; in Fig. 8, we show the energy levels in the gap for several types of interstitials $(\langle 100\rangle-$ split, bond-centered, hexagonal, tetrahedral) including $\mathrm{nn}$ relaxations for minimum $E_{\text {EHT. }}$ As can be seen, all of the highest occupied energy levels are partially filled for the neutral charge state, which allows for changes in charge state (see Sec. VI).

In Table $\mathrm{V}$, we also present the information about the electronic charge distribution (normalized to one) for each of the levels in the energy gap for the neutral charge state. These results were obtained by carrying out a Mulliken population analysis $^{50}$ which establishes the localization of the charge on the various atoms near the interstitial for each of the levels in the gap. This indicates that some of the levels correspond to localized 
defect states, whereas others are not localized. As it turns out, these nonlocalized levels are in or very close to the carrier bands, as has been discussed in more detail by Messmer and Watkins. ${ }^{1}$

\section{DISCUSSION}

We have considered the carbon interstitial in diamond using the extended Hückel theory. This theory is one of a number of semiempirical molecular-orbital theories which has been developed over the past ten years. These theories are approximations to the more complete Hartree-FockRoothaan self-consistent-field theory. Although the approximations restrict the validity, they also make possible the treatment of problems that are otherwise intractable with the more rigorous theory. We have considered the interstitial in a small portion of the diamond lattice-a model crystal-and have treated this crystal using EHT, which considers all the valence electrons of the model system. Our approach is reminiscent of the EHT treatment of configurations of organic molecules, which has been quite successful ${ }^{17}$ in predicting the most stable configuration.

The major conclusion of this paper is that on the basis of our calculations, we expect the lowestenergy configuration to be one which is interstitiallike, i.e., a split-interstitial or a bond-centered configuration; the $\langle 100\rangle$-split interstitial appears to be preferred. On the other hand, the interstitial, at the tetrahedral $(T)$ or hexagonal $(H)$ site, does not appear to be in a potential-energy mini-

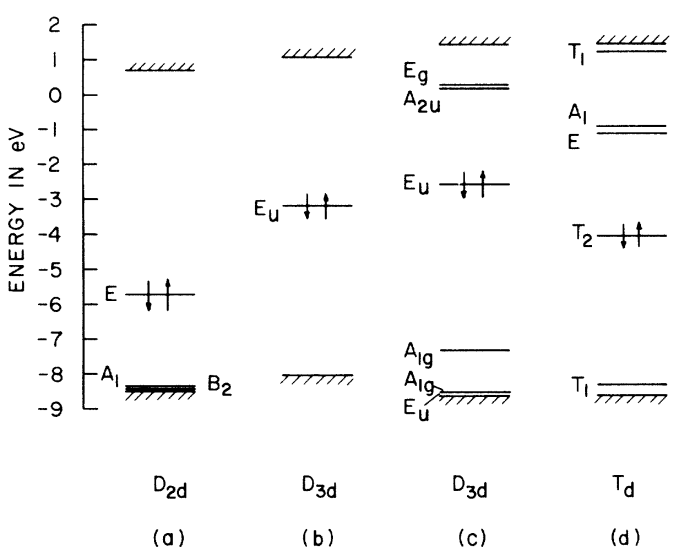

FIG. 8. One-electron MO energy levels for interstitial configurations with nearest-neighbor relaxations: (a) split - $\langle 100\rangle$ interstitial, (b) bond-centered interstitial, (c) hexagonal, and (d) tetrahedral interstitial. The point symmetry group of the interstitial configuration is given as well as the symmetry of the levels in the gap. The energy-gap edges are taken from the corresponding undistorted lattices without interstitials. Also, the occupancy of the highest filled levels is indicated.

mum and the calculated energy differences of these sites, with respect to the interstitialcies, are quite large. These results were obtained in a variety of model crystals. The results are intuitively plausible as well, since in $(T)$ or $(H)$ sites the interstitial has four broken bonds, while in the interstitialcy configurations there are only two

TABLE V. Results of EHT calculations for electronic levels in the band gap (of the undistorted lattice without interstitial) for four types of neutral carbon interstitial. The results in each case are for the relaxed configuration as described in Sec. IV. "Localized charge" means the sum of the charge density on the interstitial atoms and for the nearest neighbors.

\begin{tabular}{|c|c|c|c|c|c|c|}
\hline \multirow{2}{*}{$\begin{array}{c}\text { Site } \\
\text { (symmetry) }\end{array}$} & \multirow{2}{*}{$\begin{array}{c}\text { Level } \\
\text { symmetry }\end{array}$} & \multicolumn{5}{|c|}{ Average charge on a single atom } \\
\hline & & $E_{\mathrm{EHT}}(\mathrm{eV})$ & Interstitial & 1 st nn & 2nd nn & Localized charge \\
\hline$\langle 100\rangle$ split $^{\mathrm{a}}$ & $E^{\mathrm{b}}$ & -5.728 & 0.343 & 0.033 & 0.008 & 0.818 \\
\hline \multirow[t]{2}{*}{$\left(D_{2 d}\right)$} & $A_{1}$ & -8.355 & 0.100 & 0.040 & 0.009 & 0.360 \\
\hline & $B_{2}$ & -8.464 & 0.000 & 0.033 & 0.005 & 0.132 \\
\hline $\begin{array}{l}(B)^{\mathrm{c}} \\
\left(D_{3,}\right)\end{array}$ & $E_{u}^{\mathrm{b}}$ & -3.210 & 0.560 & 0.007 & 0.054 & 0.574 \\
\hline$(H)^{\mathrm{d}}$ & \multicolumn{6}{|c|}{$\left(D_{3 d}\right)$} \\
\hline $\begin{array}{l}(H)^{u} \\
\left(D_{0}\right)\end{array}$ & $E_{g}$ & 0.246 & 0.000 & 0.102 & 0.037 & 0.612 \\
\hline \multirow{4}{*}{$\left(D_{3 d}\right)$} & $A_{2 u}$ & 0.158 & 0.000 & 0.084 & 0.050 & 0.504 \\
\hline & $E_{u}^{\mathrm{b}}$ & -2.599 & 0.259 & 0.095 & 0.003 & 0.829 \\
\hline & $A_{1 g}$ & -7.337 & 0.729 & 0.007 & 0.016 & 0.771 \\
\hline & $A_{1 g}$ & -8.555 & 0.016 & 0.021 & 0.063 & 0.142 \\
\hline & & .80 & 0.000 & 0.024 & 0.016 & 0.144 \\
\hline$(T)$ & $A_{1}$ & -0.951 & 0.206 & 0.154 & 0.010 & 0.820 \\
\hline \multirow{3}{*}{$\left(T_{d}\right)$} & $E$ & -1.126 & 0.000 & 0.107 & 0.065 & 0.428 \\
\hline & $T_{2}{ }^{\mathrm{b}}$ & -4.097 & 0.314 & 0.104 & 0.002 & 0.736 \\
\hline & $T_{1}$ & -8.350 & 0.000 & 0.027 & 0.035 & 0.108 \\
\hline
\end{tabular}

${ }^{a}$ Results from $S 47$ lattice.

${ }^{\mathrm{b}}$ Highest occupied level. 
broken bonds. These results lead us to believe that more extensive and more rigorous calculations are not likely to overturn that conclusion.

All these considerations were made for the neutral charge state, because only for the neutral charge state can we expect a reasonably uniform charge distribution in the model crystals. As mentioned in Sec. II, a uniform charge distribution is required for EHT.

It is expected, however, that the interstitial can exist in several charged states and in this respect, it is interesting to ask what our results suggest for charged interstitial configurations. EHT yields one-electron levels, so the various charge states are obtained by filling states to a different level. The results have to be considered very cautiously, because the EHT energy levels are independent of charge state, contrary to a selfconsistent theory.

With these reservations in mind, we observe in Fig. 8 that the highest occupied levels are partially filled, i. e., they can take up or give away electrons. In Table VI we list the charge dependence of $E_{\mathrm{EHT}}$ for the $\langle 100\rangle$-split interstitial with nn relaxations and the relaxed bond-centered positionthe two lowest-energy configurations for the neutral case. As can be seen, the $\langle 100\rangle$-split interstitial has the lower energy for the singly charged states, but is higher than the relaxed bond-centered configuration for the doubly positive state.

This agrees with the suggestion made by Watkins, ${ }^{47}$ who argued that a doubly positive interstitial could find a natural position at the center of a bond.

In addition, this result of a change of the energy minimum position with charge state suggests the possibility of a low-temperature migration mechanism for the interstitial in the diamond lattice recently proposed. ${ }^{51}$ According to this Bourgoin mechanism, athermal ${ }^{52}$ migration is possible if the location of the energy minimum changes substantially with the charge state of the interstitial.

These results also constitute an attractive ex-

TABLE VI. Charge dependence of $E_{\mathrm{EHT}}$ of the two lowest-energy configurations (including $\mathrm{nn}$ relaxations) and their difference, $\Delta E_{\mathrm{EHT}}=E_{\mathrm{EHT}}$ (bond-centered interstitial) $-E_{\mathrm{EHT}}(\langle 100\rangle$ split), as calculated in the $S 47$ lattice. The sign change of $\Delta E_{E H T}$ indicates the change of equilibrium site with charge state.

\begin{tabular}{|c|c|c|c|c|}
\hline & \multicolumn{4}{|c|}{ Energy $(\mathrm{eV})$} \\
\hline & Neutral & $\begin{array}{c}\text { Singly } \\
\text { negative }\end{array}$ & $\begin{array}{c}\text { Singly } \\
\text { positive }\end{array}$ & $\begin{array}{c}\text { Doubly } \\
\text { positive }\end{array}$ \\
\hline$\langle 100\rangle$ split & -3586.25 & -3591.98 & -3580.52 & -3574.79 \\
\hline $\begin{array}{l}\text { Bond-centered } \\
\text { Interstitial }\end{array}$ & -3584.06 & -3587.93 & -3580.20 & -3576.33 \\
\hline$\Delta E_{\mathrm{EHT}}$ & 2.19 & 4.05 & 0.32 & -1.54 \\
\hline
\end{tabular}

planation for the problem of interstitial conversion in silicon. ${ }^{4}$ On the one hand, host-atom interstitials must be created as the Frenkel partner to the lattice vacancy, which is produced in a radiation damage experiment and has been detected by EPR technique $\mathrm{s}^{4,53}$; on the other hand, no direct experimental evidence is available for the interstitial yet. If we interpret $\left|\Delta E_{\mathrm{EHT}}\right|$ according to Table VI as interstitial migration energies, these barriers are quite high in terms of thermal energies for migration at $4^{\circ} \mathrm{K}$. The Bourgoin mechanism, however, supplies an athermal mechanism for the interstitial migration via the alternate capture of electrons and holes.

These results of the interstitial calculations are indeed provocative. We must remember that there are substantial limitations to the extended Hückel theory as a molecular-orbital theory. (We have already mentioned that some conclusions on the energies of different charge states cannot be made in full confidence from the EHT because it does not treat the charge self-consistently. Moreover, EHT is most successful when it treats small changes in bonding and configuration-but we have extended its application into realms of large change in bonding and configuration.) We believe the large changes in energies which are observed, and the intuitive nature of the results, lend much credence to the results.

As with any semiempirical theory, its value will depend upon its ultimate proven utility. This utility can arise in two ways. It may be useful to the theory by providing a better starting point for subsequent calculations; the work of Singhal ${ }^{49}$ is one such example. Its utility can also be gauged by its ultimate impact on experiments, and we note the initiation of a reconsideration of the mechanisms of radiation-damage production. ${ }^{54}$ As we have remarked earlier, among the many unidentified EPR spectra in silicon, none were identified with the interstitial or interstitial-related defects; we note, however, that the present results have initiated the tentative identification of an EPR spectra in diamond as being due to the interstitial ${ }^{55}$ and of EPR spectra in silicon as being due to interstitial aggregates. ${ }^{56}$

Finally, we note that there are several indications in the literature that some impurity interstitials are in interstitialcy-type configurations. For example, Watkins ${ }^{57}$ has found a low-symmetry EPR spectrum which he identifies with the boron interstitial. Channeling measurements in silicon ${ }^{58}$ also indicate that the boron is not in the tetrahedral or hexagonal site. In addition Cherki and Kalma, ${ }^{59}$ using photoconductivity, concluded that the aluminum interstitial was not at the tetrahedral site, but was much nearer a substitutional-atom site. As we will show elsewhere, comparable EHT calcu- 
lations $s^{60}$ for the boron interstitial support the view that it is in an interstitialcy-type configuration.

We conclude that both theory and experiment now suggest that the interstitial in the diamond lattice is in an interstitialcy-type configuration, i. e., either a split- $\langle 100\rangle$ interstitial (which our results favor) or a bond-centered interstitial.
*Permanent address: Physikalisches Institut der Universität, 87 Würzburg, Germany.

${ }^{\dagger}$ Research supported in part by the Office of Naval Research under Contract No. 00014-70C-0296.

${ }^{1}$ R. P. Messmer and G. D. Watkins, Phys. Rev. B 7, 2568 (1973).

${ }^{2}$ G. D. Watkins, in Proceedings of the 1972 Reading Conference on Radiation Defects in Semiconductors (Institute of Physics, London, 1973), p. 228.

${ }^{3}$ G. D. Watkins and R. P. Messmer, Phys. Rev. B 4, 2066 (1971).

${ }^{4}$ G. D. Watkins, J. Phys. Soc. Jap. Suppl. II, 22 (1963).

${ }^{5}$ J. W. Corbett, in Solid State Physics, edited by F. Seitz and

D. Turnbull (Academic, New York, 1966), Suppl. 7.

${ }^{6}$ G. D. Watkins, R. P. Messmer, C. Weigel, D. Peak, and J.

W. Corbett, Phys. Rev. Lett. 27, 1573 (1971).

${ }^{7}$ T. Yamaguchi, J. Phys. Soc. Jap. 18, 368 (1963); J. Phys. Soc. Jap. 18, 923 (1963).

${ }^{8}$ K. H. Bennemann, Phys. Rev. 137, A1497 (1965).

${ }^{9}$ R. R. Hasiguti, in Properties of Vacancies and Interstials, edited by A. D. Franklin, NBS Misc. Publ. No. 287 (U.S. GPO, Washington, D.C., 1966), p. 27.

${ }^{10}$ R. R. Hasiguti, J. Phys. Soc. Jap. 21, 1927 (1966).

${ }^{11}$ W. E. Hagston, J. Phys. Chem. Solids 3, 791 (1970).

${ }^{12}$ S. P. Singhal, Phys. Rev. B 4, 2497 (1971).

${ }^{13}$ O. Sinanoglu and K. B. Wiberg, Sigma Molecular Orbital

Theory (Yale U. P., New Haven, Conn., 1970).

${ }^{14}$ D. R. Hartree, Proc. Camb. Philos. Soc. 24, 89 (1928); Proc. Camb. Philos. Soc. 24, 111 (1928).

${ }^{15}$ V. Fock, Z. Phys. 61, 126 (1930).

${ }^{16}$ C. C. Roothaan, Rev. Mod. Phys. 23, 69 (1951).

${ }^{17}$ R. Hoffmann, J. Chem. Phys. 39, 1397 (1963).

${ }^{18}$ J. A. Pople, D. P. Santry, and G. A. Segal, J. Chem. Phys. Suppl. 43, S129 (1965).

${ }^{19}$ J. A. Pople and G. A. Segal, J. Chem. Phys. Suppl. 43, S136 (1965).

${ }^{20}$ R. P. Messmer, B. McCarroll, and C. M. Singal, J. Vac. Sci. Technol. 9, 891 (1972).

${ }^{21}$ E. B. Moore, Jr., Theor. Chim. Acta 7, 144 (1967).

${ }^{22}$ C. Hare, T. P. Sleight, W. Cooper, and G. A. Clarke, Inorg. Chem. 4, 669 (1968)

${ }^{23}$ R. P. Messmer and G. D. Watkins, Phys. Rev. Lett. 25, 656 (1970).

${ }^{24}$ L. L. Lohr, Jr., Inorg. Chem. 6, 1890 (1967).

${ }^{25}$ E. B. Moore, Jr. and C. M. Carlson, Solid State Commun. 4, 47 (1965).

${ }^{26} \mathrm{G}$. Blyholder and C. A. Coulson, Theor. Chim. Acta 10, 316 (1968).

${ }^{27}$ L. C. Allen and J. D. Russell, J. Chem. Phys. 46, 1029 (1967).

${ }^{28}$ L. C. Allen, in Ref. 13, pp. 227-248.

${ }^{29}$ L. Pauling, The Nature of the Chemical Bond (Cornell U. P., Ithaca, N. Y., 1939), p. 64.

${ }^{30}$ R. T. Sanderson, Chemical Periodicity (Reinhold, New York, 1960), p. 34.
${ }^{31}$ E. B. Moore, Jr. and C. M. Carlson, Boeing Scientific Research Laboratories Doc. No. D1-82-0649, 1967 (unpublished).

${ }^{32}$ M. D. Newton, F. P. Boer, and W. N. Lipscomb, J. Am. Chem. Soc. 88, 2353 (1966).

${ }^{33}$ E. B. Moore, Jr., in Ref. 9, p. 31.

${ }^{34}$ M. Wolfsberg and L. Helmholtz, J. Chem. Phys. 20, 873 (1952).

${ }^{35}$ R. S. Mulliken, J. Chim. Phys. 46, 497 (1949); J. Chim. Phys. 46, 675 (1949).

${ }^{36}$ J. C. Slater, Quantum Theory of Molecules and Solids (McGraw-Hill, New York, 1963), Vol. I, p. 108.

${ }^{37}$ J. Goodisman, J. Am. Chem. Soc. 91, 6552 (1969).

${ }^{38}$ R. Hoffmann and W. N. Lipscomb, J. Chem. Phys. 36, 2179 (1962); J. Chem. Phys. 36, 3489 (1962).

${ }^{39}$ R. Hoffmann and W. N. Lipscomb, J. Chem. Phys. 37, 2872 (1963).

${ }^{40}$ International Tables for X-Ray Crystallography, edited by $\mathrm{N}$. F. Henry and K. Lonsdale (Kynoch, Birmingham, England, 1969), Vol. I, p. 340.

${ }^{41}$ K. Weiser, Phys. Rev. 126, 1427 (1962).

${ }^{42}$ C. D. Clark, P. J. Dean, and P. V. Harris, Proc. R. Soc. A 277, 312 (1964).

${ }^{43}$ R. P. Messmer, Chem. Phys. Lett. 11, 589 (1971).

${ }^{44}$ R. A. Johnson and E. Brown, Phys. Rev. 127, 446 (1962)

${ }^{45}$ R. P. Messmer (unpublished results).

${ }^{46}$ See, for example, Ref. 5, p. 200.

${ }^{47}$ G. D. Watkins, as described by J. W. Corbett, in Ref. 5, p. 99.

${ }^{48} \mathrm{~J}$. Friedel, in Symposium on Radiation Effects in Semiconductor Components, edited by F. Cambou (Journees d'Electronique, Toulouse, 1967), Paper A-11.

${ }^{49}$ S. P. Singhal, Phys. Rev. B 5, 4203 (1972).

${ }^{50}$ R. S. Mulliken, J. Chem. Phys. 23, 1833 (1955).

${ }^{51}$ J. C. Bourgoin and J. W. Corbett, Phys. Lett. A 38, 135 (1972); J. C. Bourgoin, J. W. Corbett, and H. L. Frisch (unpublished).

${ }^{52}$ See also, J. S. Koehler and R. E. McKeighen, Bull. Am. Phys. Soc. 16, 396 (1971); R. E. McKeighen and J. S. Kochler, Phys. Rev. B 4, 462 (1971).

${ }^{53}$ G. D. Watkins, Radiation Damage in Semiconductors (Dunod, Paris, 1965), p. 97; G. D. Watkins, in Radiation Effects in Semiconductors, edited by F. L. Vook (Plenum, New York, 1968), p. 67.

${ }^{54} \mathrm{~J}$. W. Corbett, J. C. Bourgoin, and C. Weigel, in Ref. 2, p. 1

${ }^{55}$ Y. M. Kim, Y. H. Lee, P. R. Brosious, and J. W. Corbett, in Ref. 2, p. 202.

${ }^{56}$ Y. H. Lee, Y. M. Kim, and J. W. Corbett, Radiat. Eff. 15, 77 (1972).

${ }^{57}$ G. D. Watkins, in Ref. 48, Paper A-1.

${ }^{58}$ J. C. North and W. M. Gibson, Appl. Phys. Lett. 16, 126 (1970).

${ }^{59}$ M. Cherki and A. H. Kalma, Phys. Rev. B 1, 647 (1970).

${ }^{60}$ C. Weigel, D. Peak, J. W. Corbett, G. D. Watkins, and R. P. Messmer (unpublished). 